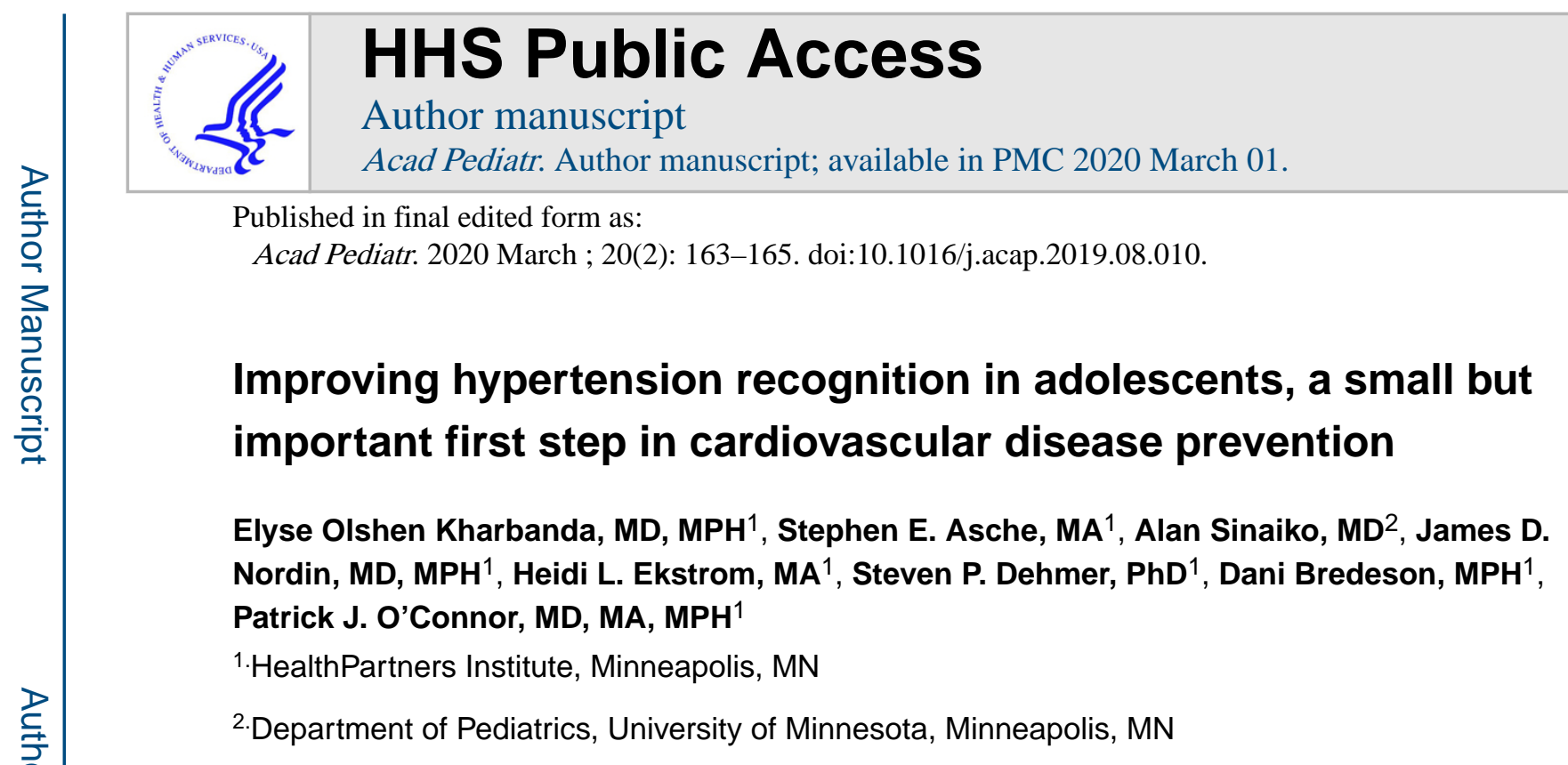

Keywords

Decision support; hypertension; adolescents

Blood pressure $(\mathrm{BP})$ is routinely measured during pediatric primary care visits. It is estimated that $1-3 \%$ of youth in primary care settings meet clinical criteria for HT and an additional $5 \%$ to $10 \%$ have isolated BP measurements in the hypertensive range. ${ }^{1,2}$ Elevated BP and HT during childhood and adolescence, although generally asymptomatic, is associated with immediate adverse health effects, including left ventricular hypertrophy (LVH). ${ }^{3}$ More importantly, HT in youth tracks into adulthood, and predicts long-term cardiovascular morbidity and mortality. ${ }^{4}$

For children up to 12 years of age HT is defined as having BP recorded at three or more visits at or above the $95^{\text {th }}$ percentile. Based on the updated, 2017 BP guidelines, for youth 13-17 years, the threshold for HT is $130 / 80 \mathrm{mmHg} .{ }^{5}$ As children average one to two primary care visits each year, over time there should be sufficient patient BP data stored in the electronic health record (EHR) to identify HT. However, our group and others have demonstrated that due to lack of familiarity with current pediatric BP guidelines, competing priorities at primary care visits, and the complexities of age, sex and height-based BP percentile calculations, elevated BP and HT are frequently not clinically recognized.

Clinical decision support (CDS), integrated within an existing electronic health record (EHR) platform and delivered at the point of care, is ideally suited to address barriers to elevated BP and HT recognition. Automated calculations of BP percentiles for current and prior visits can reduce the time needed to identify an incident or persistently elevated BP. Computer prompts regarding BP classification, recommended evaluations, and timing for follow-up can assist providers unfamiliar with pediatric BP guidelines.

Address correspondence to: Elyse Olshen Kharbanda, MD, MPH, Senior Investigator, HealthPartners Institute, 8170 33rd Ave South, Mail stop 23301A, Minneapolis, MN 55425.

Conflict of Interest: The authors have no conflicts of interest to disclose.

Clinical Trial Registration: 
We previously developed, piloted, and refined an EHR-linked CDS to improve the recognition and initial management of elevated BP and HT in youth 10-17 years of age. ${ }^{6}$ The impact of this novel CDS on recognition and next steps in care was then formally assessed in a two-year cluster-randomized trial, conducted in 20 primary care clinics within our large integrated health system. ${ }^{6}$ As described in the Jan-Feb 2018 issue of Academic Pediatrics, using data from the first year of the trial, we identified approximately 1200 adolescents with an incident or a first elevated BP, evenly divided between intervention and usual care clinics. As compared to those attending usual care clinics, patients with elevated $\mathrm{BP}$ recorded at visits to CDS intervention clinics were more likely to have their elevated BP remeasured during the visit and were more likely to be diagnosed with elevated BP.

Although pediatric BP guidelines recommend that patients with elevated BP return for an additional measurement in 1-2 weeks, rates for repeat BP measurement within 30 days were low and did not differ significantly among those at CDS intervention clinics. ${ }^{7}$

More recently we have described results from the full trial. Of 31,579 patients $10-17$ years of age with one or more BPs measured over a two-year period at study clinics, $1.8 \%$ met clinical criteria for HT. Within 6 months of meeting criteria for incident HT, 54.9\% of patients attending CDS clinics versus $21.3 \%$ of patients attending usual care clinics had their HT clinically recognized $(\mathrm{p}<0.001) .{ }^{8}$ Using diagnosis codes alone to identify recognition, the rates were $47 \%$ and $14 \%$, respectively. HT recognition was strongly associated with lifestyle referral or provider counseling regarding exercise and weight loss. A majority of providers surveyed at the end of the intervention thought that the CDS was useful in identifying patients with elevated BP or hypertension, $94 \%$ agreed that time using the CDS was "time well-spent" and 95\% agreed that the CDS tool was useful for shared decisionmaking. ${ }^{8}$ In an accompanying 2018 editorial, our CDS tool was described as "the most comprehensive and well-integrated pediatric BP CDS described to date." 9 In a separate economic evaluation [submitted to Academic Pediatrics concurrently with this submission], we found that among patients with incident HT, availability of the CDS at their primary care clinical encounter was not associated with significant increases in medical expenditures over 12-months of follow-up.

Given the demonstrated effectiveness of the CDS in the initial trial, along with the positive feedback from providers using the tool, with support from our health system clinical leadership we have expanded the targeted age range to ages 6-17 years, updated algorithms to account for new definitions based on the 2017 pediatric BP guidelines ${ }^{5}$ and simplified the CDS, for use outside of a research study. In June 2018, following provider and staff training we implemented the CDS, now known as Peds \& TeenBP, for use in 36 primary care clinics in Minnesota and Western Wisconsin (including 20 from the initial trial.) Over a 4-month period following this implementation, there were 29,028 visits among 6-17 year olds with a BP measured. Of these, 3305 (11.3\%) had an elevated BP and $48 \%$ of elevated BPs were remeasured at the visit. Over the 4-month period, 171 patients $(0.6 \%)$ newly met criteria for HT and about one-third were diagnosed with HT or elevated BP within 6 months of meeting criteria. (Table 1) Of note, there is no ongoing training or audit-feedback to further support CDS use. As of February 2019, the Peds \&TeenBPCDS is live in all 55 primary care clinics and 19 subspecialty clinics serving children within our large integrated care system. Future directions include plans to implement this effective CDS in new health systems, with a focus 
on rural and other settings where access to pediatricians and pediatric subspecialists may be limited.

The short term benefits of CDS such as the Peds \& TeenBP, improving the quality of BP measurement through repeated measurement of an initial elevated BP and increasing clinical recognition of elevated BP and HT are clear. Identification or clinical recognition of HT is an important and necessary first step. Nevertheless, the long term benefits of diagnosing HT in youth, including the potential for early recognition to delay or prevent cardiovascular disease, has been identified as a gap in evidence and priority for ongoing pediatric hypertension ressearch. ${ }^{10}$

\section{Funding Source:}

All phases of this study were supported the National Institute of Health (R01 HL115082 [to EOK]). The sponsor was not involved in the study design, data collection, analysis or interpretation of data, writing of the manuscript or the decision to submit for publication.

\section{References}

1. Koebnick C, Black MH, Wu J, et al. The prevalence of primary pediatric prehypertension and hypertension in a real-world managed care system. J Clin Hypertens (Greenwich). 2013;15(11):784-792. [PubMed: 24283596]

2. Lo JC, Sinaiko A, Chandra M, et al. Prehypertension and hypertension in community-based pediatric practice. Pediatrics. 2013;131(2):e415-424. [PubMed: 23359583]

3. Zhang T, Li S, Bazzano L, He J, Whelton P, Chen W. Trajectories of Childhood Blood Pressure and Adult Left Ventricular Hypertrophy: The Bogalusa Heart Study. Hypertension. 2018;72(1):93-101. [PubMed: 29785961]

4. Du T, Fernandez C, Barshop R, Chen W, Urbina EM, Bazzano LA. 2017 Pediatric Hypertension Guidelines Improve Prediction of Adult Cardiovascular Outcomes. Hypertension. 2019:HYPERTENSIONAHA11812469.

5. Flynn JT, Kaelber DC, Baker-Smith CM, et al. Clinical Practice Guideline for Screening and Management of High Blood Pressure in Children and Adolescents. Pediatrics. 2017;140(3).

6. Kharbanda EO, Nordin JD, Sinaiko AR, et al. TeenBP: Development and Piloting of an EHRLinked Clinical Decision Support System to Improve Recognition of Hypertension in Adolescents. EGEMS (Wash DC). 2015;3(2):1142. [PubMed: 26290886]

7. Kharbanda EO, Asche SE, Sinaiko A, et al. Evaluation of an Electronic Clinical Decision Support Tool for Incident Elevated BP in Adolescents. Acad Pediatr. 2018;18(1):43-50. [PubMed: 28723587]

8. Kharbanda EO, Asche SE, Sinaiko AR, et al. Clinical Decision Support for Recognition and Management of Hypertension: A Randomized Trial. Pediatrics. 2018;141(2).

9. Pollack AH, Flynn JT. You Can’t Treat a Problem if You Don't Recognize It. Pediatrics. 2018;141(2).

10. Taylor-Zapata P, Baker-Smith CM, Burckart G, et al. Research Gaps in Primary Pediatric Hypertension. Pediatrics. 2019;143(5). 


\section{What's new}

Electronic health record linked clinical decision support can help to increase clinical recognition of elevated blood pressure and hypertension in youth, improving the quality of blood pressure measurement and promoting guideline adherent care. 
Table 1.

Data on recognition of elevated BP and hypertension in 36 primary care clinics using Peds \& TeenBP, July 1 , 2018 - October 31, 2018

\begin{tabular}{|l|c|}
\hline & $\mathbf{N}=\mathbf{2 4 , 5 3 2}$ unique patients \\
Male & $\mathrm{N}(\%)$ \\
Female & $12,294(50.1)$ \\
6-12 years & $12,237(49.9)$ \\
13-17 years & $14,086(57.4)$ \\
Asian & $10,446(42.6)$ \\
African American & $2202(9.0)$ \\
Native American & $4958(20.2)$ \\
White & $328(1.3)$ \\
Unknown / other & $14,268(58.2)$ \\
Hispanic & $2776(11.3)$ \\
& $1745(7.1)$ \\
Initial BP at visit elevated ${ }^{*}$ & $\mathbf{N = 2 9 , 0 2 8 ~ v i s i t s ~ w i t h ~ B P ~ m e a s u r e d ~}$ \\
Of 3305 visits with initial BP elevated, BP repeated at visit & $\mathrm{N}(\%)$ \\
Lowest BP at visit elevated * & $3305(11.3)$ \\
Met criteria for incident hypertension ** & 1587 (48.0) \\
Of 171 patients meeting criteria for hypertension, hypertension or elevated BP diagnosed within 6 months & $55(32.1)$ \\
\hline
\end{tabular}

For youth 6-12 years, systolic BP or diastolic BP $\geq 95^{\text {th }}$ percentile, using 2017 Guideline thresholds; for youth 13-17 years, systolic BP $\geq 130$ $\mathrm{mmHg}$ or diastolic BP $\$ 80 \mathrm{mmHg}$

**

Elevated BP at 3 or more visits, without prior hypertension diagnosis

$\mathrm{BP}=$ blood pressure 